\title{
Mechanism to explore lamination rate
}

\author{
Lionel Rossi* \\ Department of Aeronautics, Imperial College London, Prince Consort Road, London SW7 2AZ, United Kingdom
}

(Received 15 June 2009; published 9 February 2010)

\begin{abstract}
A mechanism amenable to laminate and fold flows is identified and quantified. This laminating mechanism follows from a physical and experimental approach relying on the interlaced structure of velocity and Lagrangian acceleration. The Lagrangian acceleration being the resultant of the forces applied on particle fluids, the component of acceleration perpendicular to the velocity vector allows the quantification of a rate of change of the velocity's direction, i.e., the local angular Lagrangian velocity, $\dot{\theta}$. The spatial variation in $\dot{\theta}$ is then used to predict and measure the lamination and folding rate. To support and illustrate this approach, three basic experimental flows, driven by electromagnetic forces, are discussed and compared. Folding rate intensities are extracted for different characteristic length scales. Also, good agreement is found between grid deformation and the prediction of lamination rate. This quantification of lamination rate opens new avenues for the design of mixers, in particular at low Reynolds numbers.
\end{abstract}

DOI: 10.1103/PhysRevE.81.027301

Mixing is an important phenomenon in numerous natural and industrial flows. Large scale mixers often rely on the intrinsic properties of turbulence to stir and consequently mix flows. When the scales are reduced, e.g., for microflows, the flow's stirring cannot rely on intrinsic properties of turbulence [1]. Such flows need to be engineered [2], so as to mix elements of very low diffusivity. For a given energy, efficient stirrers increase the length of interfaces in a minimum of time or length of the microchannel. Consequently, standard mixing approaches rely on the study of regions with high values of strain as they are amenable to produce fast and local exponential stretching. This has led to the separation of flows in hyperbolic and elliptic domains (OkuboWeiss decomposition) and the study of: the distribution of hyperbolic stagnation points, strain persistence and maps, finite-time Lyapunov exponents, e.g., [3-9]. As introduced by the seminal talk of Reynolds [10], proper combinations of lamination and stretching, like a baker folding and stretching pastry, can also lead to an exponential growth of material lines and consequently interfaces $[6,11]$. While, alone, shear or lamination would lead to linear growth. Figure 1 illustrates the exponential growth of a colored band with the repetition of stretching-laminating cycles. In addition, once laminated/striated structures enter a domain of high strain (hyperbolic domain) they are stretched and compressed. This enhances (locally) mixing by extending the length of interfaces and importantly by increasing concentration gradients with the reduction of the striation thickness, as illustrated in Fig. 2(b). Recently, Bajer et al. [12] showed that the lamination, due to differential velocities, increases the diffusion in the center of analytical vortices. Similarly, the analytical whorl illustrated in Fig. 3(b) highlights a higher lamination where the derivative of the whorl's angular velocity along its radius is the highest, as shown by the red (dark gray) curve. Such spirals laminate flows by rolling material lines. Lamination is also used in circular devices such as the one illustrated in Fig. 3(a) which has been applied to microfluidic [13]. To date, the quantification of laminating and folding

\footnotetext{
*1.rossi@imperial.ac.uk; rossi_lionel@yahoo.fr
}

\section{PACS number(s): 47.51.+a, 47.63.mf, 47.85.1k}

mechanisms is still largely unexplored. Folding is often related to the need for flows stretched in confined domains to bend. Also, bending and corresponding striation can appear within local jets, as illustrated by Fig. 2(a). Even if chaos and folding could be quantified, a posteriori, using Horseshoe maps [14] and geometrical quantification of the interface pattern [15], the quantification of folding often relies on qualitative visualizations, e.g., [16-18]. The terminology folding and lamination rate represents here, broad sense, the rate at which a material line is bent and/or rolled so as to generate striated patterns. Lamination rate is a key element to complement mixing studies and contribute to the design of new mixers, e.g., ordered devices at low Reynolds numbers [19]. This letter attempts to isolate and to quantify a mechanism to explore lamination rates.

The proposed fundamental mechanism quantifies the action of the forces applied on a material line to turn it at different angular speeds. The Lagrangian acceleration, a, represents the force exerted on a particle-fluid by its environment and governs the alteration in direction and intensity of its Lagrangian velocity, $\mathbf{u}_{\mathbf{L}}$. This is described using the Navier Stokes equation,

$$
\mathbf{a}=\frac{\partial \mathbf{u}_{\mathbf{L}}}{\partial t}=\frac{\partial \mathbf{u}}{\partial t}+(\mathbf{u} \cdot \nabla) \mathbf{u}=-\frac{1}{\rho} \nabla P+\nu \Delta \mathbf{u}+\frac{1}{\rho} \mathbf{f},
$$

where $\mathbf{u}$ is the velocity of the flow at the spatial position $\mathbf{x}$ $\left[\mathbf{u}_{\mathbf{L}}(t)=\mathbf{u}\left(\mathbf{x}_{\mathbf{E}}, t\right)\right.$ when $\mathbf{x}_{\mathbf{E}}$ is the spatial position of the fluid

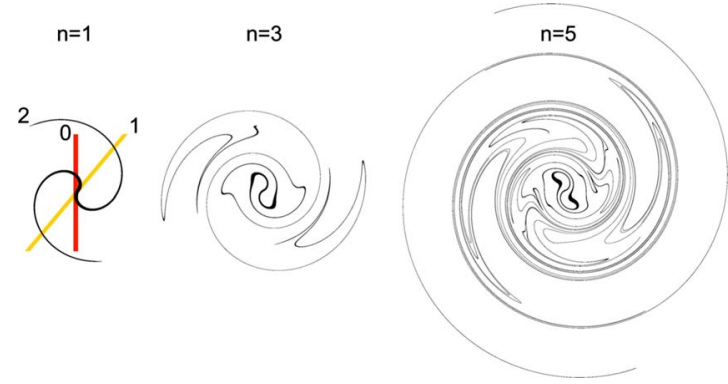

FIG. 1. (Color online) Stretching of a colored band via cyclic combinations of shear and lamination. The building of these cycles is illustrated for the first iteration, $n=1$. 


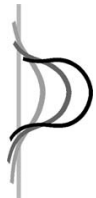

(a)

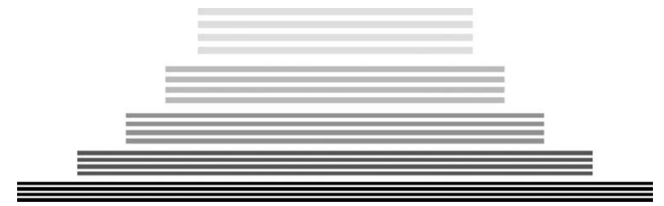

(b)

FIG. 2. (a) Bending of a material line. (b) Evolution of a striated structure in a pure strain region. Temporal evolution from light gray to black.

element]; $-\frac{1}{\rho} \nabla P, \nu \Delta \mathbf{u}$, and $\frac{1}{\rho} \mathbf{f}$ are, respectively, the actions of pressure, viscous, and body forces.

This new approach considers the alignment between the velocity and the Lagrangian acceleration, and in particular the component of the acceleration perpendicular to the velocity, $\mathbf{a}_{\mathbf{p}}=\mathbf{a}-\mathbf{a}_{\mathbf{t}}$ with $\mathbf{a}_{\mathbf{t}}=(\mathbf{a} \cdot \mathbf{u}) \mathbf{u} /(\mathbf{u} \cdot \mathbf{u})$, to determine the local Lagrangian angular velocity, $\dot{\theta}$, (i.e., the rate of change of direction of the Lagrangian velocity vector at position $\mathbf{x}$ ) around the rotation vector, $\mathbf{e}_{\mathbf{f}}$; see Eq. (2). It should be noted that $\left(\mathbf{u} /\|\mathbf{u}\|, \mathbf{a}_{\mathbf{p}} /\left\|\mathbf{a}_{\mathbf{p}}\right\|, \mathbf{e}_{\mathbf{f}}\right)$ defines a Frenet frame attached to the fluid element, e.g., [20]. Also, while being mathematically related, $\dot{\theta}^{2}=\kappa^{2} \mathbf{u} \cdot \mathbf{u}$, the Lagrangian angular velocity differs from the trajectory curvature $(\kappa)$ which has been the focus of recent studies, e.g., [20].

$$
\dot{\theta}=\sqrt{\frac{\mathbf{a}_{\mathbf{p}} \cdot \mathbf{a}_{\mathbf{p}}}{\mathbf{u} \cdot \mathbf{u}}}, \quad \mathbf{e}_{\mathbf{f}}=\frac{\mathbf{u} \times \mathbf{a}_{\mathbf{p}}}{\left\|\mathbf{u} \times \mathbf{a}_{\mathbf{p}}\right\|} .
$$

The folding and lamination rate of a material line element, $\mathbf{d} \ell$, around the direction $\mathbf{e}_{\mathbf{f}}$ is now defined using the spatial variation in $\dot{\theta}$ along $\mathbf{d} \ell$. This is also an elegant way to characterize the differential winding or twisting of linked twist map [11]. The matrix of folding and lamination rate, $\mathbf{G}_{\theta}$, around the direction $\mathbf{e}_{\mathbf{f}}$ at position $\mathbf{x}$ is defined by Eq. (3) which considers the Eulerian derivatives of the Lagrangian quantity $\dot{\theta}$.

$$
\mathbf{G}_{\theta}[i, j]=\delta_{i j} \frac{\partial \dot{\theta}(\mathbf{x})}{\partial x_{i}} .
$$

The intensity of the lamination and folding rate, $\dot{\mathcal{R}}_{\text {fol }}$, applied to $\mathbf{d} \ell$ is then quantified using Eq. (4),

$$
\dot{\mathcal{R}}_{f o l}(\mathbf{d} \ell)=\left\|\mathbf{G}_{\dot{\theta}} \mathbf{d} \ell\right\| .
$$

When the spatial variation in the Lagrangian angular velocity is coherent and sustained over a given domain, the

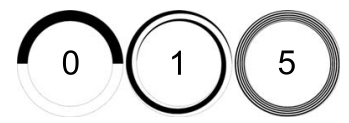

(a) (b)

FIG. 3. (Color online) Illustrations of Lamination. (a) Circular device: the internal disk rotates while the external boundary is immobile. (b) Lamination within a whorl of angular velocity $\dot{\theta}$ $=\dot{\theta}_{0} \exp \left(1-(r / R)^{2}\right)$ after six revolutions. The black and red (gray) lines respectively illustrate the whorl and $\partial \dot{\theta} / \partial r$.

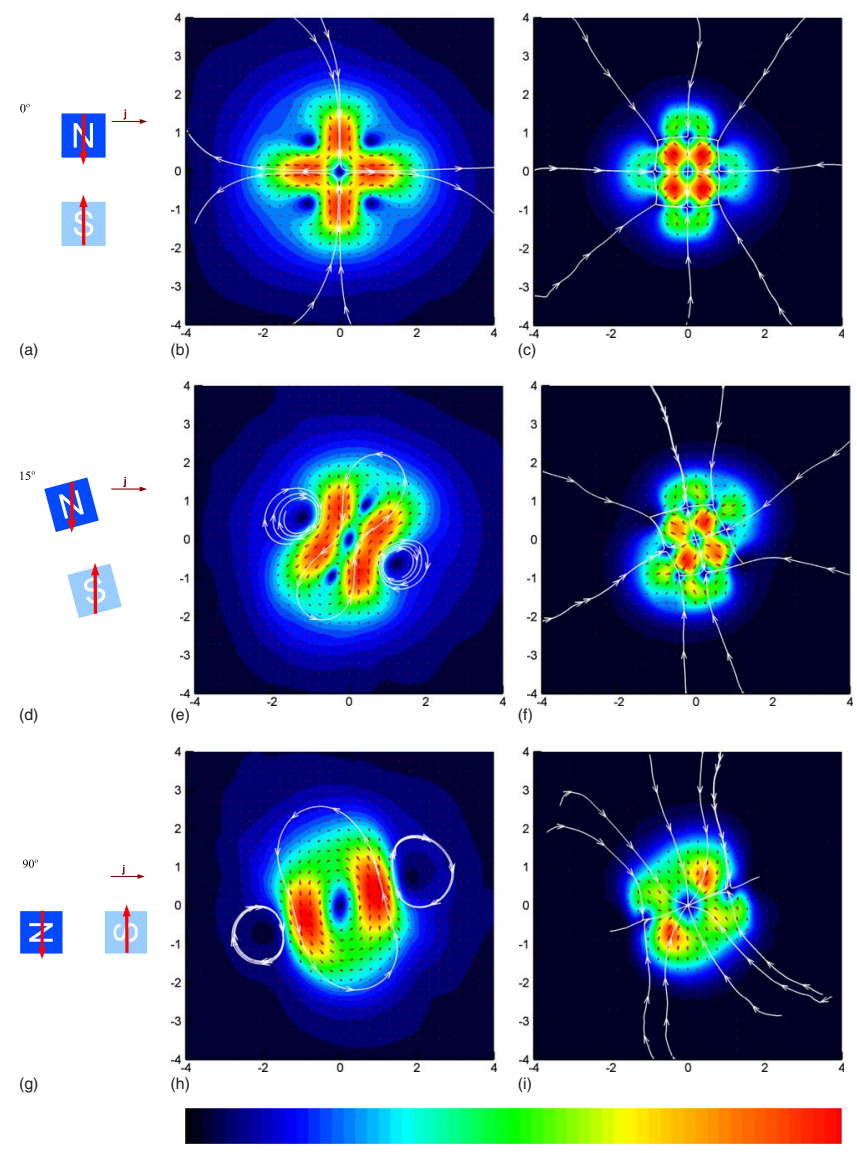

FIG. 4. (Color online) (a) Schematics of the different forcing configurations. The arrows indicate the direction of the electrical current $\mathbf{j}$ and of the main forces pumping the flow. (b) Velocity fields, $\mathbf{u}^{*}$. (c) Acceleration fields, $\mathbf{a}^{*}$. Color scales minima (black) are 0 , maxima are 4.5 for (b) and 6.5 for (c)

striations generated within this domain increase with its size. To extract the laminating potential of flows (different from the effective lamination of given material lines) at a given length scale, $\ell$, the folding rate intensity given by Eq. (4) is simplified to Eq. (5) where $\nabla \dot{\theta}$ should be coarse grained at size $\ell$.

$$
\dot{\mathcal{R}}_{f o l}=\|\nabla \dot{\theta}\| \ell .
$$

This mechanism applies to whorls, tendrils and any flow structures as it is by definition local in time and space. It is the persistence and coherence in time and in space that would indicate the type of laminated structures generated. Also, this definition relies on the choice of a frame of reference which by default could be taken as the laboratory/mixer reference or the mean velocity.

The proposed mechanism is now applied to experimental flows using a shallow layer of brine driven by electromagnetic forces, similarly to $[7,9,21]$. Fundamental flow configurations are driven by electromagnetic forces, $\mathbf{f}$, so as to design the geometry of the flows [19]. To achieve this, a pair of square permanent magnets $\left(L_{M}=40 \mathrm{~mm}\right)$ is used with different orientations compared to the direction of the electrical current. The flow configurations are consequently named 


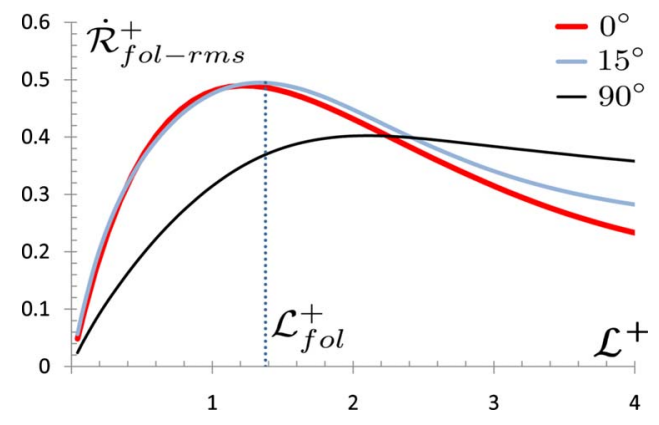

FIG. 5. (Color online) Lamination rate intensity, $\dot{\mathcal{R}}_{\text {fol-rms }}^{+}$vs $\mathcal{L}^{+}$.

according to this angle, as illustrated in the first column of Fig. 4. The corresponding flows are laminar, quasi-twodimensional and quasisteady for the low forcing intensity considered [19]. The experimental data are well resolved in space and time. The length of the PIV (particle image velocimetry) correlation windows is more than ten times smaller than the magnets' length with an overlap of $50 \%$. The temporal resolution allows real-time PIV between consecutive pictures of a continuous sequence. This enables the extraction of the spatial distribution of the velocity and acceleration fields via an hybrid PIV-PTVA (particule tracking velocimetry and accelerometry) procedure. For more details see [19,22,23]. These electromagnetically driven flows present different geometries and topologies for both velocity, $\mathbf{u}^{*}=\mathbf{u} / u_{r m s}$, and acceleration fields, $\mathbf{a}^{*}=\mathbf{a} / a_{r m s}$, as illustrated by Fig. 4 . rms denotes the root mean square values computed over the measurement domain. The values obtained for configurations $0^{\circ}, 15^{\circ}, 90^{\circ}$ are, respectively, 1.73, 1.88, and $1.98 \mathrm{~mm} / \mathrm{s}$ for $u_{r m s}$ and $0.291,0.297$, and $0.265 \mathrm{~mm} / \mathrm{s}^{2}$ for $a_{r m s}$. The reference scales of the forcing are chosen as: $L_{M}$ for the length, $u_{r e f}=\sqrt{\frac{f_{r m s} L_{M}}{2 \rho}} \simeq 0.015 \mathrm{~m} / \mathrm{s}$ for the velocity, $\quad a_{r e f}=\frac{1}{\rho} f_{r m s} \simeq 0.003 \mathrm{~m} / \mathrm{s}^{2}$ for the acceleration, $\dot{\mathcal{R}}_{\text {fol-ref }}=a_{\text {ref }} / u_{\text {ref }} \simeq 0.19 \mathrm{rad} / \mathrm{s}$ for the lamination rate
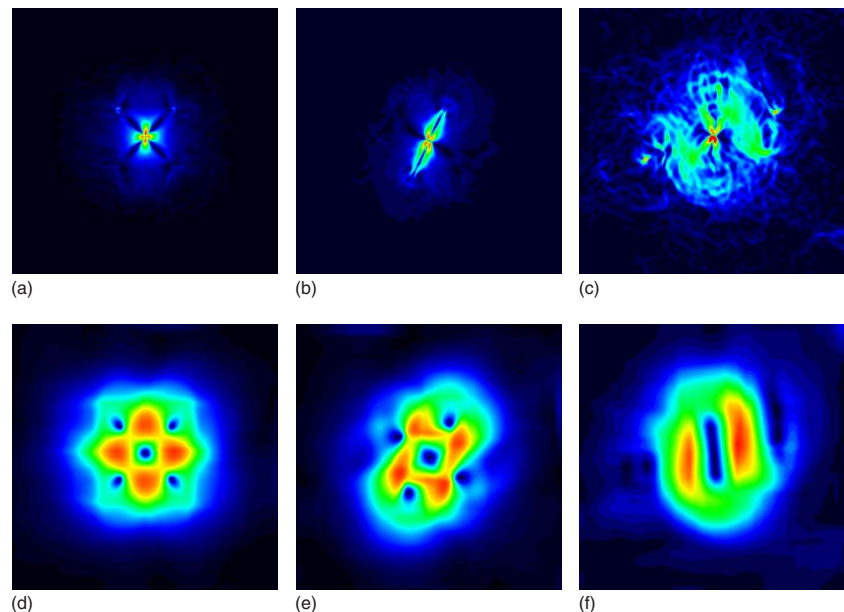

FIG. 6. (Color online) Lamination rate intensities, $\dot{\mathcal{R}}_{\text {fol }}^{+}$, for the three flow configurations and the two length scales: (a) mesh size, $\mathcal{L}^{+}=0.086$, (b) $\mathcal{L}_{\text {fol }}^{+}=1.38$. Same domain and color scale as Fig. 4. Color scale minima (black) are zero, maxima are (by row from top left) $0.4,0.4,0.04,0.9,0.9,0.4$.
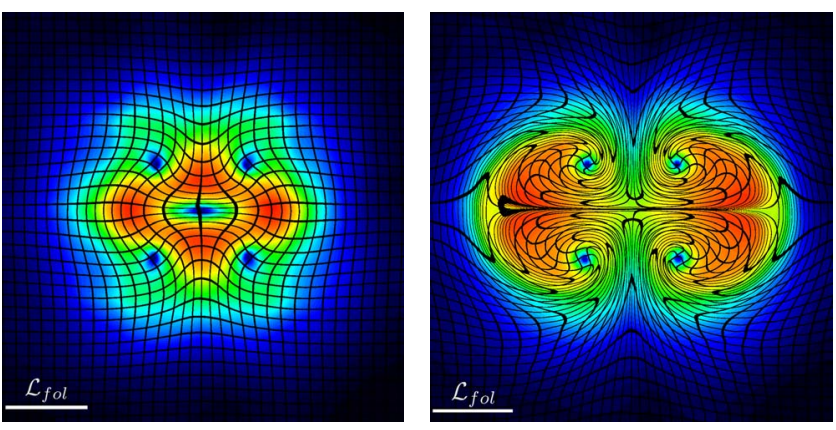

(a)

(b)

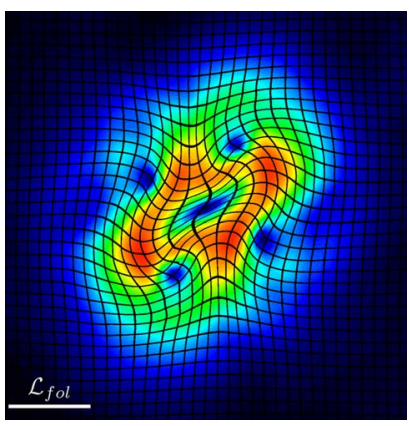

(c)

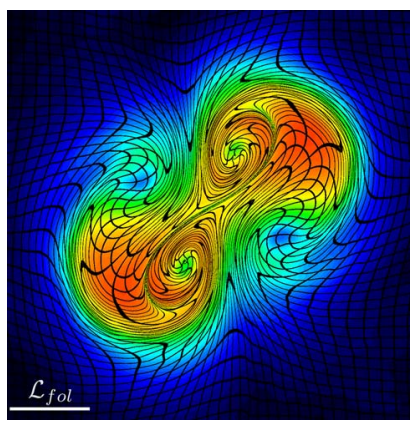

(d)

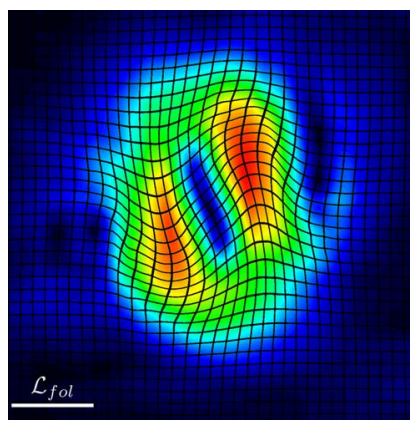

(e)

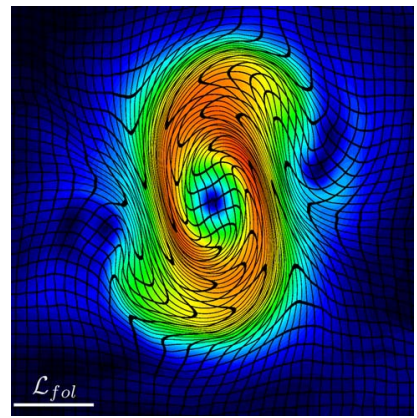

(f)

FIG. 7. (Color online) Deformation of a grid superposed to maps representing the mean (over time) lamination rate along particle fluids tracked backward in time. Left column corresponds to $t^{+} \simeq 0.61$ and right column to $t^{+}=4.9 . \mathcal{L}_{\text {fol }}^{+}=1.38$. Same color scale as Fig. 4, minima (black) are zero and maxima, by row from top left, are: $0.9,0.6,0.9,0.6,0.4,0.3$.

and $t_{r e f}=L_{M} / u_{r e f} \simeq 5.2 \mathrm{~s}$ for the time. Consequently, $\dot{\mathcal{R}}_{\text {fol-rms }}^{+}=\dot{\mathcal{R}}_{\text {fol-rms }} / \dot{\mathcal{R}}_{\text {fol-ref }}, \mathcal{L}^{+}=\mathcal{L} / L_{M}$, and $t^{+}=t / t_{\text {ref }}$.

The laminating rates of these three typical flow configurations are now quantified. Under practical consideration, the smallest accessible $\ell$ is the size of the PIV mesh; $\ell=\ell_{m}$. For length scales larger than $\ell_{m}$, the value of $\nabla \dot{\theta}\left(\mathbf{e}_{\mathbf{f}} \cdot \mathbf{z}\right)$, where $\mathbf{z}$ is the direction perpendicular to the measurement plane $\left(\mathbf{e}_{\mathbf{f}} \cdot \mathbf{z}\right.$ $= \pm 1$, according to the direction of rotation) is coarse grained by averaging within mobile windows of size $\mathcal{L}$. The laminating rate intensity distribution is thus computed with $\ell=\mathcal{L}$. Figure 5 gives the evolution of the root mean square of the folding rate intensity, $\dot{\mathcal{R}}_{\text {fol-rms }}^{+}$, versus $\mathcal{L}^{+}$. $\dot{\mathcal{R}}_{\text {fol-rms }}^{+}$ reaches maxima values for typical length scales which are much higher (one decade) than the folding rate at the mesh size. This indicates that these laminating flow structures are 
coherent and more efficient around a typical length-scale. For the configuration $15^{\circ}$, this length scale is noted $\mathcal{L}_{\text {fol }}$ with $\mathcal{L}_{\text {fol }}^{+}=1.38$. Also, the configuration $90^{\circ}$ has a lower lamination rate than the two other configurations for sizes smaller than approximately $\mathcal{L}^{+}=2$ which is the spacing between the magnet centers and the characteristic lamination length scale of case $90^{\circ}$. The spatial distributions of $\dot{\mathcal{R}}_{\text {fol-rms }}^{+}$given in Fig. 6 for $\ell=\ell_{m}$ and $\ell=\mathcal{L}_{\text {fol }}$ complement Fig. 5. For the three orientations larger magnitudes are observed over larger domains for $\ell=\mathcal{L}_{f o l}$.

Finally, the lamination of a flow is an integrative process along the Lagrangian trajectories of the particles defining a material line. To highlight the ability of the proposed definition to quantify the laminating properties of flows, $\dot{\mathcal{R}}_{\text {fol }}^{+}$is averaged, over a time $t$, along fluid element trajectories tracked backward in time. The initial positions are chosen as the PIV mesh to build the corresponding maps of lamination. This roughly quantifies the distribution and intensity of the lamination within the flows after given times. The final test is performed by comparing flow visualizations (starting at $-t$ with a regular distribution of black bands) with these mean quantities. If the measure of $\dot{\mathcal{R}}_{f o l}^{+}$is coherent, the laminated domains should be found where the backward integration is high. Figure 7 presents superposed results at a short $\left(t^{+}\right.$ $=0.61)$ and a long $\left(t^{+}=4.9 \sim L_{M} / u_{r m s}\right)$ tracking time with $\ell$ $=\mathcal{L}_{f o l}$. It should be mentioned that the mean of the Lagrangian lamination rate fields is roughly conserved during backward integration. Also, this mean is within a few $\%$ of the mean lamination rate. After a short time, and for the three configurations, the mesh is bent where the maxima of the mean lamination rate are found. This captures some bending properties of the local "jets" observed in Fig. 4. In fact, such identification relies on the acceleration mechanisms shaping these local jets. After a more significant time, the three con- figurations show good agreement between the flow visualizations and the spatial distribution of the mean lamination rate. For $0^{\circ}$, the maximum lamination is observed where the mean of $\dot{\mathcal{R}}_{f o l}^{+}$is maximum. The four circles with small integral values superpose with domains where the flow has been weakly folded as shown by the persistence of the straight crosses. For $15^{\circ}$, the eight shape of the visualization pattern superposes well with the map. The local minima of the integration noticeable within both loops of the eight, correspond to a weaker lamination of the visualization pattern and the local maxima correspond to material lines which have been bent and/or rolled. Finally, in the $90^{\circ}$ case, the heart of the flow appears like in a solid body rotation and consequently the lamination is very weak there and the main folding is found around this heart.

The experimental results presented in this letter support the proposed mechanism to explore, quantify and predict lamination and folding rates of flows and mixers. The proposed definition can be applied to a broad variety of flows and studies. The quantification and prediction of folding and lamination rate should be a key element for the design of mixers and should open avenues for the constructive design of multicycles mixers. The experimental results confirm that such an approach based on the combined distribution of velocity and Lagrangian acceleration can now be pursued. Indeed, the recent possibility for experimentalists to access the spatial distribution of the Lagrangian acceleration supports this new approach. Further proof, discussion, and applications to unsteady flows should come from the author's group and readers.

The author acknowledges The Royal Society, the EPSRC, G. Fishpool, J.M. Garcia de la Cruz, S. Lardeau, and EPSRC Grant No. EP/D072034/1.
[1] T. M. Squires and S. R. Quake, Rev. Mod. Phys. 77, 977 (2005).

[2] J. M. Ottino and S. Wiggins, Science 305, 485 (2004).

[3] E. Dresselhaus and M. Tabor, J. Fluid Mech. 236, 415 (1992).

[4] G. Haller, Chaos 10, 99 (2000).

[5] G. Haller and G. Yuan, Physica D 147, 352 (2000).

[6] J. M. Ottino,The Kinematics of Mixing: Stretching, Chaos, and Transport (Cambridge University Press, Cambridge, England, 1989).

[7] L. Rossi, J. C. Vassilicos, and Y. Hardalupas, Phys. Rev. Lett. 97, 144501 (2006).

[8] D. Elhmaïdi, A. Provenzale, and A. Babiano, J. Fluid Mech. 257, 533 (1993).

[9] G. A. Voth, G. Haller, and J. P. Gollub, Phys. Rev. Lett. 88, 254501 (2002).

[10] O. Reynolds, On Study of Fluid Motion by Means of Coloured Bands, in Notices of the Proceedings at the Meetings of the Members of the Royal Institution of Great Britain, with Abstracts of the Discourses Delivered at the Evening Meetings, Vol. XIV 1893-1895 (William Clowes and Sons, London,1893), p. 129.

[11] S. Wiggins and J. M. Ottino, Philos. Trans. R. Soc. London, Ser. A 362, 937 (2004).
[12] K. Bajer, A. P. Bassom, and A. D. Gilbert, J. Fluid Mech. 437, 395 (2001).

[13] S. R. Quake and A. Scherer, Science 290, 1536 (2000).

[14] J. M. Ottino, S. C. Jana, and V. S. Chakravarthy, Phys. Fluids 6, 685 (1994).

[15] P. Tabeling, M. Chabert, A. Dodge, C. Jullien, and F. Okkels, Philos. Trans. R. Soc. London 362, 987 (2004).

[16] A. D. Stroock, S. K. W. Dertinger, A. Ajdari, I. Mezic, H. A. Stone, and G. M. Whitesides, Science 295, 647 (2002).

[17] T. Cubaud and T. G. Mason, Phys. Rev. Lett. 96, 114501 (2006).

[18] J. Frederick R. Phelan, N. R. Hughes, and J. A. Pathak, Phys. Fluids 20, 023101 (2008).

[19] L. Rossi, S. Bocquet, S. Ferrari, J. M. Garcia de la Cruz, and S. Lardeau, Int. J. Heat Fluid Flow 30, 505 (2009).

[20] W. Braun, F. De Lillo, and B. Eckhardt, J. Turbul. 7, N62 (2006).

[21] M.-C. Jullien, P. Castiglione, and P. Tabeling, Phys. Rev. Lett. 85, 3636 (2000).

[22] S. Ferrari and L. Rossi, Exp. Fluids 44, 873 (2008).

[23] S. Lardeau, S. Ferrari, and L. Rossi, Phys. Fluids 20, 127101 (2008). 